PROCEEDINGS OF THE

AMERICAN MATHEMATICAL SOCIETY

Volume 135, Number 5, May 2007, Pages 1319-1327

S 0002-9939(06)08664-3

Article electronically published on December 27, 2006

\title{
ON THE FINITENESS PROPERTIES OF EXTENSION AND TORSION FUNCTORS OF LOCAL COHOMOLOGY MODULES
}

\author{
KAZEM KHASHYARMANESH \\ (Communicated by Bernd Ulrich)
}

\begin{abstract}
Let $R$ be a commutative Noetherian ring with non-zero identity, $\mathfrak{a}$ and $\mathfrak{b}$ ideals of $R$ with $\mathfrak{a} \subseteq \mathfrak{b}$, and $M$ a finitely generated $R$-module. In this paper, for fixed integers $j$ and $n$, we study the finiteness of $\operatorname{Ext}_{R}^{j}\left(R / \mathfrak{b}, H_{\mathfrak{a}}^{n}(M)\right)$ and $\operatorname{Tor}_{j}^{R}\left(R / \mathfrak{b}, H_{\mathfrak{a}}^{n}(M)\right)$ in several cases.
\end{abstract}

\section{INTRODUCTION}

Throughout this paper, $R$ will denote a commutative Noetherian ring with nonzero identity and $\mathfrak{a}$ an ideal of $R$. Also $M$ will denote a finitely generated $R$-module. Our terminology follows the textbook [3] on local cohomology.

It is a well-known result that if $R$ is a complete local ring with maximal ideal $\mathfrak{m}$, then the $R$-module $M$ is Artinian if and only if $\operatorname{Supp}_{R}(M) \subseteq\{\mathfrak{m}\}$ and $\operatorname{Ext}_{R}^{i}(R / \mathfrak{m}, M)$ is finitely generated for all $i \in \mathbb{N}_{0}$ (cf. [9, Proposition 1.1]). (We use $\mathbb{N}_{0}$ (respectively $\mathbb{N}$ ) to denote the set of non-negative (respectively positive) integers.) The following conjecture was made by Grothendieck (see [8, Expose XIII, Conjecture $1.2])$.

1.1. For any ideal $\mathfrak{a}$ and any finitely generated $R$-module, the module

$$
\operatorname{Hom}_{R}\left(R / \mathfrak{a}, H_{\mathfrak{a}}^{n}(M)\right)
$$

is finitely generated for all $n \geqslant 0$, where $H_{\mathfrak{a}}^{n}(M)$ is the $n$-th local cohomology module of $M$ with respect to $\mathfrak{a}$.

Hartshorne has produced in [9] a counterexample which shows that this conjecture is false even when $R$ is regular. Hartshorne asked the following question.

1.2. If $\mathfrak{a}$ is an ideal of $R$ and $M$ is a finitely generated $R$-module, when are $\operatorname{Ext}_{R}^{j}\left(R / \mathfrak{a}, H_{\mathfrak{a}}^{n}(M)\right)$ finitely generated for all $n$ and $j$ ?

Hartshorne defined a module $N$ to be a-cofinite if the support of $N$ is contained in $V(\mathfrak{a})$ and $\operatorname{Ext}_{R}^{j}(R / \mathfrak{a}, N)$ is finitely generated for all $j \in \mathbb{N}_{0}$. By working in the

Received by the editors November 2, 2005 and, in revised form, February 2, 2006.

2000 Mathematics Subject Classification. Primary 13D45, 13D07.

Key words and phrases. Local cohomology modules, extension functor, torsion functor, cofinite modules, associated primes, cohomological dimension, coassociated primes, filter regular sequences.

The author was partially supported by a grant from the Institute for Studies in Theoretical Physics and Mathematics (IPM) Iran (No. 84130025).

(C)2006 American Mathematical Society Reverts to public domain 28 years from publication 
derived category, he also showed that if $M$ is a finitely generated $R$-module, where $R$ is a complete regular local ring, then $H_{\mathfrak{a}}^{n}(M)$ is a-cofinite in two cases:

(i) $\mathfrak{a}$ is a non-zero principal ideal 9 , Corollary 6.3];

(ii) $\mathfrak{a}$ is a prime ideal with dimension one [9, Corollary 7.7].

There are several papers devoted to the extension of Hartshorne's second result to more general situations: We refer the reader to the papers of Huneke and Koh [10], Delfino 4], Delfino and Marley [5], and Yoshida [19].

In view of (1.2), we consider the following questions.

1.3. If $\mathfrak{a}$ and $\mathfrak{b}$ are ideals of $R$ and $M$ is a finitely generated $R$-module, for fixed integers $j$ and $n$, when is $\operatorname{Ext}_{R}^{j}\left(R / \mathfrak{b}, H_{\mathfrak{a}}^{n}(M)\right)$ finitely generated?

1.4. If $\mathfrak{a}$ and $\mathfrak{b}$ are ideals of $R$ and $M$ is a finitely generated $R$-module, for fixed integers $j$ and $n$, when is $\operatorname{Tor}_{j}^{R}\left(R / \mathfrak{b}, H_{\mathfrak{a}}^{n}(M)\right)$ finitely generated?

One of the main results in this paper is the following theorem.

Theorem 1.5. Fix $j \in \mathbb{N}_{0}, n \in \mathbb{N}$, the ideals $\mathfrak{a}$ and $\mathfrak{b}$ with $\mathfrak{a} \subseteq \mathfrak{b}$, and a finitely generated $R$-module $M$ of dimension d. Assume that

(i) $\operatorname{Ext}_{R}^{j+t+1}\left(R / \mathfrak{b}, H_{\mathfrak{a}}^{n-t}(M)\right)$ is finitely generated for $t=1, \ldots, n$, and

(ii) $\operatorname{Ext}_{R}^{j-k-1}\left(R / \mathfrak{b}, H_{\mathfrak{a}}^{n+k}(M)\right)$ is finitely generated for $k=1, \ldots, d-n$.

Then $\operatorname{Ext}_{R}^{j}\left(R / \mathfrak{b}, H_{\mathfrak{a}}^{n}(M)\right)$ is finitely generated.

Also, we show that generalizations of theorems in [6, Theorem 2.1], [7, Theorem A and Theorem B], [1, Theorem 1.2], 13, Proposition 2.5], 2, Theorem 2.2] and [12, Theorem $\mathrm{B}(\beta)$ ] are immediate consequences from the above theorem. Note that Proposition 2.5 in 13 and Theorem 1.2 in [1] were proved by using spectral sequences. As we point out in Remark 3.4, the proof of Theorem 2.1 in [6] contains some flaws, but we will show that the statement of Theorem 2.1 in $[$ is true (see Corollary 3.5(i)). Moreover, we can establish an analogue of the above results for $\operatorname{Tor}_{j}^{R}\left(R / \mathfrak{b}, H_{\mathfrak{a}}^{n}(M)\right)$.

\section{EXACT SEQUENCES OF LOCAL COHOMOLOGY MODULES}

The concept of a filter regular sequence plays an important role in this paper. We say that a sequence $x_{1}, \ldots, x_{n}$ of elements of $\mathfrak{a}$ is an $\mathfrak{a}$-filter regular sequence on $M$, if

$$
\operatorname{Supp}_{R}\left(\frac{\left(x_{1}, \ldots, x_{i-1}\right) M:_{M} x_{i}}{\left(x_{1}, \ldots, x_{i-1}\right) M}\right) \subseteq V(\mathfrak{a})
$$

for all $i=1, \ldots, n$, where $V(\mathfrak{a})$ denotes the set of prime ideals of $R$ containing $\mathfrak{a}$. Also, we say that an element $x \in \mathfrak{a}$ is an $\mathfrak{a}$-filter regular element on $M$ if $\operatorname{Supp}_{R}\left(0:_{M} x\right) \subseteq V(\mathfrak{a})$. The concept of an $\mathfrak{a}$-filter regular sequence on $M$ is a generalization of the concept of a filter regular sequence which has been studied in [15, [16] and has led to some interesting results. Both concepts coincide if $\mathfrak{a}$ is an $\mathfrak{m}$-primary ideal of a local ring with maximal ideal $\mathfrak{m}$. Note that $x_{1}, \ldots, x_{n}$ is a weak $M$-sequence if and only if it is an $R$-filter regular sequence on $M$. It is easy to see that the analogue of [16, Appendix 2(ii)] holds true whenever $R$ is Noetherian, $M$ is finitely generated and $\mathfrak{m}$ is replaced by $\mathfrak{a}$, so that, if $x_{1}, \ldots, x_{n}$ is an $\mathfrak{a}$-filter regular sequence on $M$, then there is an element $y \in \mathfrak{a}$ such that $x_{1}, \ldots, x_{n}, y$ is an $\mathfrak{a}$-filter regular sequence on $M$. Thus, for any positive integer $n$, there exists an $\mathfrak{a}$-filter regular sequence on $M$ of length $n$ (also see [1, Proposition 2.2]). 
Proposition 2.1 (See [11, Proposition 1.2] and 1, Proposition 2.3]). Let $x_{1}, \ldots, x_{n}$ $(n>0)$ be an $\mathfrak{a}$-filter regular sequence on $M$. Then there are the following isomorphisms:

$$
H_{\mathfrak{a}}^{i}(M) \cong \begin{cases}H_{\left(x_{1}, \ldots, x_{n}\right)}^{i}(M) & \text { for } 0 \leq i<n, \\ H_{\mathfrak{a}}^{i-n}\left(H_{\left(x_{1}, \ldots, x_{n}\right)}^{n}(M)\right) & \text { for } n \leq i .\end{cases}
$$

The most important technical part is the following result.

Proposition 2.2. Let $M$ be a finitely generated $R$-module and let $\mathfrak{a}, \mathfrak{b}$ be ideals of $R$ with $\mathfrak{a} \subseteq \mathfrak{b}$. Then

(i) for any $\mathfrak{a}$-filter regular element $x$ on $M$, there exist a long exact sequence

$$
\begin{aligned}
0 & \longrightarrow \operatorname{Ext}_{R}^{1}\left(R / \mathfrak{b}, H_{\mathfrak{a}}^{0}(M)\right) \longrightarrow \operatorname{Ext}_{R}^{1}(R / \mathfrak{b}, M) \longrightarrow \operatorname{Hom}_{R}\left(R / \mathfrak{b}, H_{(x)}^{1}(M)\right) \\
& \longrightarrow \operatorname{Ext}_{R}^{2}\left(R / \mathfrak{b}, H_{\mathfrak{a}}^{0}(M)\right) \longrightarrow \operatorname{Ext}_{R}^{2}(R / \mathfrak{b}, M) \longrightarrow \operatorname{Ext}_{R}^{1}\left(R / \mathfrak{b}, H_{(x)}^{1}(M)\right) \\
& \longrightarrow \\
& \longrightarrow \operatorname{Ext}_{R}^{j}\left(R / \mathfrak{b}, H_{\mathfrak{a}}^{0}(M)\right) \longrightarrow \operatorname{Ext}_{R}^{j}(R / \mathfrak{b}, M) \longrightarrow \operatorname{Ext}_{R}^{j-1}\left(R / \mathfrak{b}, H_{(x)}^{1}(M)\right) \\
& \longrightarrow \operatorname{Ext}_{R}^{j+1}\left(R / \mathfrak{b}, H_{\mathfrak{a}}^{0}(M)\right) \longrightarrow \cdots \\
& \text { and the isomorphism }
\end{aligned}
$$

$$
\operatorname{Hom}_{R}\left(R / \mathfrak{b}, H_{\mathfrak{a}}^{0}(M)\right) \cong \operatorname{Hom}_{R}\left(R / \mathfrak{b}, H_{(x)}^{1}(M)\right) \text {, and }
$$

(ii) for any positive integer $n$ and any $\mathfrak{a}$-filter regular sequence $x_{1}, \ldots, x_{n+1} \in \mathfrak{a}$ on $M$, there exist a long exact sequence

$$
\begin{aligned}
0 \longrightarrow & \operatorname{Ext}_{R}^{1}\left(R / \mathfrak{b}, H_{\mathfrak{a}}^{n}(M)\right) \longrightarrow \operatorname{Ext}_{R}^{1}\left(R / \mathfrak{b}, H_{I}^{n}(M)\right) \longrightarrow \operatorname{Hom}_{R}\left(R / \mathfrak{b}, H_{J}^{n+1}(M)\right) \\
\longrightarrow & \operatorname{Ext}_{R}^{2}\left(R / \mathfrak{b}, H_{\mathfrak{a}}^{n}(M)\right) \longrightarrow \operatorname{Ext}_{R}^{2}\left(R / \mathfrak{b}, H_{I}^{n}(M)\right) \longrightarrow \operatorname{Ext}_{R}^{1}\left(R / \mathfrak{b}, H_{J}^{n+1}(M)\right) \\
\longrightarrow & \cdots \\
\longrightarrow & \operatorname{Ext}_{R}^{j}\left(R / \mathfrak{b}, H_{\mathfrak{a}}^{n}(M)\right) \longrightarrow \operatorname{Ext}_{R}^{j}\left(R / \mathfrak{b}, H_{I}^{n}(M)\right) \longrightarrow \operatorname{Ext}_{R}^{j-1}\left(R / \mathfrak{b}, H_{J}^{n+1}(M)\right) \\
\longrightarrow & \operatorname{Ext}_{R}^{j+1}\left(R / \mathfrak{b}, H_{\mathfrak{a}}^{n}(M)\right) \longrightarrow \cdots \\
& \text { and the isomorphism }
\end{aligned}
$$

$$
\operatorname{Hom}_{R}\left(R / \mathfrak{b}, H_{\mathfrak{a}}^{n}(M)\right) \cong \operatorname{Hom}_{R}\left(R / \mathfrak{b}, H_{J}^{n}(M)\right),
$$

where $I:=\left(x_{1}, \ldots, x_{n}\right)$ and $J:=\left(x_{1}, \ldots, x_{n+1}\right)$.

Proof. Let $n \in \mathbb{N}_{0}$ and $x_{1}, \ldots, x_{n+1} \in \mathfrak{a}$ be an $\mathfrak{a}$-filter regular sequence on $M$. (Note that the existence of such a sequence is explained in the beginning of this section.) Put $U_{0}:=M$ and $U_{i}:=H_{\left(x_{1}, \ldots, x_{i}\right)}^{i}(M)$ for $i=1, \ldots, n+1$. Note that $x_{1}, \ldots, x_{i}$ is an $\left(x_{1}, \ldots, x_{i+1}\right)$-filter regular sequence on $M$. Then, in view of [3, Exercises 1.1.2 and 2.1.4] and Proposition 2.1, we have

$$
\begin{aligned}
H_{\left(x_{i+1}\right)}^{0}\left(U_{i}\right) & \cong H_{\left(x_{i+1}\right)}^{0}\left(H_{\left(x_{1}, \ldots, x_{i}\right)}^{0}\left(U_{i}\right)\right) \cong H_{\left(x_{1}, \ldots, x_{i+1}\right)}^{0}\left(U_{i}\right) \\
& \cong H_{\left(x_{1}, \ldots, x_{i+1}\right)}^{i}(M) \cong H_{\mathfrak{a}}^{i}(M) \text { and } \\
H_{\left(x_{i+1}\right)}^{1}\left(U_{i}\right) & \cong H_{\left(x_{1}, \ldots, x_{i+1}\right)}^{1}\left(U_{i}\right) \cong H_{\left(x_{1}, \ldots, x_{i+1}\right)}^{i+1}(M)
\end{aligned}
$$

So, by [3, Remark 2.2.17], for each $i=0,1, \ldots, n$, we obtain the following exact sequence:

$$
0 \longrightarrow H_{\mathfrak{a}}^{i}(M) \longrightarrow U_{i} \stackrel{f_{i}}{\longrightarrow}\left(U_{i}\right)_{x_{i+1}} \longrightarrow U_{i+1} \longrightarrow 0
$$


Put $L_{i}:=\operatorname{Im} f_{i}$ for $i=0,1, \ldots, n$. Since the multiplication by $x_{i+1}$ is an automorphism on $\left(U_{i}\right)_{x_{i+1}}$ and $x_{i+1} \in \mathfrak{b}$, it follows from the exact sequence

$$
0 \longrightarrow L_{i} \longrightarrow\left(U_{i}\right)_{x_{i+1}} \longrightarrow U_{i+1} \longrightarrow 0
$$

that

$$
\begin{aligned}
\operatorname{Hom}_{R}\left(R / \mathfrak{b}, L_{i}\right) & =0 & & (*) \quad \text { and } \\
\operatorname{Ext}_{R}^{j}\left(R / \mathfrak{b}, L_{i}\right) & \cong \operatorname{Ext}_{R}^{j-1}\left(R / \mathfrak{b}, U_{i+1}\right) & & (* *)
\end{aligned}
$$

for all $i=0,1, \ldots, n$ and $j \in \mathbb{N}$. Hence, for $i=0,1, \ldots, n$, by applying the functor $\operatorname{Hom}_{R}(R / \mathfrak{b},-)$ on the exact sequence

$$
0 \longrightarrow H_{\mathfrak{a}}^{i}(M) \longrightarrow U_{i} \longrightarrow L_{i} \longrightarrow 0,
$$

in conjunction with $(*)$ and $(* *)$, one can obtain an exact sequence

$$
\begin{aligned}
& 0 \longrightarrow \operatorname{Ext}_{R}^{1}\left(R / \mathfrak{b}, H_{\mathfrak{a}}^{i}(M)\right) \longrightarrow \operatorname{Ext}_{R}^{1}\left(R / \mathfrak{b}, U_{i}\right) \longrightarrow \operatorname{Hom}_{R}\left(R / \mathfrak{b}, U_{i+1}\right) \\
& \longrightarrow \operatorname{Ext}_{R}^{2}\left(R / \mathfrak{b}, H_{\mathfrak{a}}^{i}(M)\right) \longrightarrow \operatorname{Ext}_{R}^{2}\left(R / \mathfrak{b}, U_{i}\right) \longrightarrow \operatorname{Ext}_{R}^{1}\left(R / \mathfrak{b}, U_{i+1}\right) \\
& \longrightarrow \cdots \\
& \longrightarrow \operatorname{Ext}_{R}^{j}\left(R / \mathfrak{b}, H_{\mathfrak{a}}^{i}(M)\right) \longrightarrow \operatorname{Ext}_{R}^{j}\left(R / \mathfrak{b}, U_{i}\right) \longrightarrow \operatorname{Ext}_{R}^{j-1}\left(R / \mathfrak{b}, U_{i+1}\right) \\
& \longrightarrow \operatorname{Ext}_{R}^{j+1}\left(R / \mathfrak{b}, H_{\mathfrak{a}}^{i}(M)\right) \longrightarrow \cdots
\end{aligned}
$$

and the isomorphism

$$
\operatorname{Hom}_{R}\left(R / \mathfrak{b}, H_{\mathfrak{a}}^{i}(M)\right) \cong \operatorname{Hom}_{R}\left(R / \mathfrak{b}, U_{i}\right) .
$$

Now, the results follow from the cases $i=n=0$ and $i=n>0$.

By making a straightforward modification to the arguments in the proof of Proposition 2.2, one can obtain the following proposition.

Proposition 2.3. Let $M$ be a finitely generated $R$-module and let $\mathfrak{a}, \mathfrak{b}$ be ideals of $R$ with $\mathfrak{a} \subseteq \mathfrak{b}$. Then

(i) for any $\mathfrak{a}$-filter regular element $x$ on $M$, there exists a long exact sequence

$$
\begin{aligned}
& R / \mathfrak{b} \otimes_{R} H_{\mathfrak{a}}^{0}(M) \longrightarrow R / \mathfrak{b} \otimes_{R} M \longrightarrow \operatorname{Tor}_{1}^{R}\left(R / \mathfrak{b}, H_{(x)}^{1}(M)\right) \\
& \operatorname{Tor}_{1}^{R}\left(R / \mathfrak{b}, H_{\mathfrak{a}}^{0}(M)\right) \longrightarrow 0 \\
& \operatorname{Tor}_{1}^{R}(R / \mathfrak{b}, M) \longrightarrow \operatorname{Tor}_{2}^{R}\left(R / \mathfrak{b}, H_{(x)}^{1}(M)\right) \\
& \cdots \longrightarrow \\
& \cdots \longrightarrow \operatorname{Tor}_{j-1}^{R}(R / \mathfrak{b}, M) \\
& \cdots \longrightarrow \operatorname{Tor}_{j}^{R}\left(R / \mathfrak{b}, H_{(x)}^{R}\left(R / \mathfrak{b}, H_{(x)}^{1}(M)\right)\right. \\
& \cdots \longrightarrow
\end{aligned}
$$

and $R / \mathfrak{b} \otimes_{R} H_{(x)}^{1}(M)=0$, and

(ii) for any positive integer $n$ and any $\mathfrak{a}$-filter regular sequence $x_{1}, \ldots, x_{n+1} \in \mathfrak{a}$ on $M$, there exists a long exact sequence

$$
\begin{aligned}
& R / \mathfrak{b} \otimes_{R} H_{\mathfrak{a}}^{n}(M) \longrightarrow R / \mathfrak{b} \otimes_{R} H_{I}^{n}(M) \longrightarrow \operatorname{Tor}_{1}^{R}\left(R / \mathfrak{b}, H_{J}^{n+1}(M)\right) \\
& \operatorname{Tor}_{1}^{R}\left(R / \mathfrak{b}, H_{\mathfrak{a}}^{n}(M)\right) \longrightarrow \operatorname{Tor}_{1}^{R}\left(R / \mathfrak{b}, H_{I}^{n}(M)\right) \longrightarrow \operatorname{Tor}_{2}^{R}\left(R / \mathfrak{b}, H_{J}^{n+1}(M)\right) \\
& \cdots \longrightarrow \\
& \operatorname{Tor}_{j-1}^{R}\left(R / \mathfrak{b}, H_{\mathfrak{a}}^{n}(M)\right) \longrightarrow \operatorname{Tor}_{j-1}^{R}\left(R / \mathfrak{b}, H_{I}^{n}(M)\right) \longrightarrow \operatorname{Tor}_{j}^{R}\left(R / \mathfrak{b}, H_{J}^{n+1}(M)\right) \longrightarrow \\
& \cdots \longrightarrow \operatorname{Tor}_{j+1}^{R}\left(R / \mathfrak{b}, H_{J}^{n+1}(M)\right) \longrightarrow \\
& \text { and } R / \mathfrak{b} \otimes_{R} H_{J}^{n+1}(M)=0, \text { where } I:=\left(x_{1}, \ldots, x_{n}\right) \text { and } J:=\left(x_{1}, \ldots, x_{n+1}\right) .
\end{aligned}
$$




\section{Finiteness PROPERTIES OF EXTENSION FUNCTORS OF LOCAL COHOMOLOGY MODULES}

Let $\mathfrak{a}$ and $\mathfrak{b}$ be ideals of $R$ with $\mathfrak{a} \subseteq \mathfrak{b}$. In this section, we are interested in conditions which ensure that, for fixed integers $j$ and $n, \operatorname{Ext}_{R}^{j}\left(R / \mathfrak{b}, H_{\mathfrak{a}}^{n}(M)\right)$ is finitely generated. To this end, in the first lemma, for a fixed $j \in \mathbb{N}_{0}$, we determine conditions on $H_{\mathfrak{a}}^{i}(M)(i<n)$ which ensure that $\operatorname{Ext}_{R}^{j}\left(R / \mathfrak{a}, H_{\left(x_{1}, \ldots, x_{n}\right)}^{n}(M)\right)$ is finitely generated for every a-filter regular sequence $x_{1}, \ldots, x_{n}$ on $M$. Moreover, in the second lemma, we are interested in seeking conditions on $H_{\mathfrak{a}}^{i}(M)(i>n)$ which again are sufficient for the finiteness of $\operatorname{Ext}_{R}^{j}\left(R / \mathfrak{b}, H_{\left(x_{1}, \ldots, x_{n}\right)}^{n}(M)\right)$, for every a-filter regular sequence $x_{1}, \ldots, x_{n}$ on $M$. Note that, in general, $H_{\left(x_{1}, \ldots, x_{n}\right)}^{n}(M)$ is not finitely generated, unless $H_{\left(x_{1}, \ldots, x_{n}\right)}^{i}(M)=0$ for all $i>0$ (cf. [19, Proposition $3.1])$.

Lemma 3.1. Let $\mathfrak{a}, \mathfrak{b}$ be ideals of $R$ with $\mathfrak{a} \subseteq \mathfrak{b}$. Let $n \in \mathbb{N}$ and $j \in \mathbb{N}_{0}$ be fixed integers such that $\operatorname{Ext}_{R}^{j+t+1}\left(R / \mathfrak{b}, H_{\mathfrak{a}}^{n-t}(M)\right)$ is finitely generated for all $t=1, \ldots, n$. Then, for any $\mathfrak{a}$-filter regular sequence $x_{1}, \ldots, x_{n}$ on $M, \operatorname{Ext}_{R}^{j}\left(R / \mathfrak{b}, H_{\left(x_{1}, \ldots, x_{n}\right)}^{n}(M)\right)$ is finitely generated.

Proof. Let $x_{1}, \ldots, x_{n}$ be an $\mathfrak{a}$-filter regular sequence on $M$. We use induction on $n$, the length of the sequence. When $n=1$, the result is immediate from Proposition 2.2(i). Assume, inductively, that $n \geqslant 2$, and the result has been proved for positive integers smaller than $n$. By the inductive hypothesis, $\operatorname{Ext}_{R}^{j+1}\left(R / \mathfrak{b}, H_{\left(x_{1}, \ldots, x_{n-1}\right)}^{n-1}(M)\right)$ is finitely generated. Also, in view of Proposition 2.2(ii), there exists a long exact sequence

$$
\begin{aligned}
\cdots \longrightarrow \operatorname{Ext}_{R}^{j+1}\left(R / \mathfrak{b}, H_{\left(x_{1}, \ldots, x_{n-1}\right)}^{n-1}(M)\right) & \longrightarrow \operatorname{Ext}_{R}^{j}\left(R / \mathfrak{b}, H_{\left(x_{1}, \ldots, x_{n}\right)}^{n}(M)\right) \\
& \longrightarrow \operatorname{Ext}_{R}^{j+2}\left(R / \mathfrak{b}, H_{\mathfrak{a}}^{n-1}(M)\right) \longrightarrow \cdots
\end{aligned}
$$

Now, the result follows from the assumption that $\operatorname{Ext}_{R}^{j+2}\left(R / \mathfrak{b}, H_{\mathfrak{a}}^{n-1}(M)\right)$ is finitely generated.

Lemma 3.2. Let $M$ be a finitely generated $R$-module of dimension d. Let $n \in \mathbb{N}$ and $j \in \mathbb{N}_{0}$ be fixed integers such that $\operatorname{Ext}_{R}^{j-k+1}\left(R / \mathfrak{b}, H_{\mathfrak{a}}^{n+k-1}(M)\right)$ is finitely generated for all $k=1, \ldots, d-n+1$. Then, for any $\mathfrak{a}$-filter regular sequence $x_{1}, \ldots, x_{n}$ on $M, \operatorname{Ext}_{R}^{j}\left(R / \mathfrak{b}, H_{\left(x_{1}, \ldots, x_{n}\right)}^{n}(M)\right)$ is finitely generated.

Proof. By the Grothendieck vanishing theorem (cf. [3, Theorem 6.1.2]), we can assume that $n \leqslant d$. Let $x_{1}, \ldots, x_{n}$ be an $\mathfrak{a}$-filter regular sequence on $M$. Then there exists $x_{n+1} \in \mathfrak{a}$ such that $x_{1}, \ldots, x_{n+1}$ is an $\mathfrak{a}$-filter regular sequence on $M$. We use (descending) induction on $n$. When $n=d$, in view of Proposition 2.2(ii) and [3. Theorem 6.1.2], $\operatorname{Ext}_{R}^{j}\left(R / \mathfrak{b}, H_{\left(x_{1}, \ldots, x_{d}\right)}^{d}(M)\right)$ is a homomorphic image of $\operatorname{Ext}_{R}^{j}\left(R / \mathfrak{b}, H_{\mathfrak{a}}^{d}(M)\right)$ and, by our assumption, the latter module is finitely generated. Assume, inductively that $0 \leqslant n<d$ and the result has been proved for integers greater than $n$. By inductive hypothesis, $\operatorname{Ext}_{R}^{j-1}\left(R / \mathfrak{b}, H_{\left(x_{1}, \ldots, x_{n+1}\right)}^{n+1}(M)\right)$ is finitely generated. Moreover, in view of Proposition 2.2(ii), there exists an exact sequence

$$
\begin{aligned}
\cdots \longrightarrow \operatorname{Ext}_{R}^{j}\left(R / \mathfrak{b}, H_{\mathfrak{a}}^{n}(M)\right) & \longrightarrow \operatorname{Ext}_{R}^{j}\left(R / \mathfrak{b}, H_{\left(x_{1}, \ldots, x_{n}\right)}^{n}(M)\right) \\
& \longrightarrow \operatorname{Ext}_{R}^{j-1}\left(R / \mathfrak{b}, H_{\left(x_{1}, \ldots, x_{n+1}\right)}^{n+1}(M)\right) \longrightarrow \cdots
\end{aligned}
$$


The result now follows from the assumption that $\operatorname{Ext}_{R}^{j}\left(R / \mathfrak{b}, H_{\mathfrak{a}}^{n}(M)\right)$ is finitely generated.

Now we are ready to present one of our main results.

Theorem 3.3. Fix $j \in \mathbb{N}_{0}, n \in \mathbb{N}$, the ideals $\mathfrak{a}$ and $\mathfrak{b}$ with $\mathfrak{a} \subseteq \mathfrak{b}$, and a finitely generated $R$-module $M$ of dimension $d$. Assume that

(i) $\operatorname{Ext}_{R}^{j+t+1}\left(R / \mathfrak{b}, H_{\mathfrak{a}}^{n-t}(M)\right)$ is finitely generated for all $t=1, \ldots, n$, and

(ii) $\operatorname{Ext}_{R}^{j-k-1}\left(R / \mathfrak{b}, H_{\mathfrak{a}}^{n+k}(M)\right)$ is finitely generated for all $k=1, \ldots, d-n$.

Then $\operatorname{Ext}_{R}^{j}\left(R / \mathfrak{b}, H_{\mathfrak{a}}^{n}(M)\right)$ is finitely generated.

Proof. Let $x_{1}, \ldots, x_{n+1} \in \mathfrak{a}$ be an $\mathfrak{a}$-filter regular sequence on $M$. By Lemma 3.1, condition (i) implies that $\operatorname{Ext}_{R}^{j}\left(R / \mathfrak{b}, H_{\left(x_{1}, \ldots, x_{n}\right)}^{n}(M)\right)$ is finitely generated. Also, in view of Lemma 3.2, our hypothesis in condition (ii) ensures that

$$
\operatorname{Ext}_{R}^{j-2}\left(R / \mathfrak{b}, H_{\left(x_{1}, \ldots, x_{n+1}\right)}^{n+1}(M)\right)
$$

is finitely generated. Next, in view of Proposition 2.2(ii), we have the following exact sequence:

$$
\begin{aligned}
\operatorname{Ext}_{R}^{j-2}\left(R / \mathfrak{b}, H_{\left(x_{1}, \ldots, x_{n+1}\right)}^{n+1}(M)\right) & \longrightarrow \operatorname{Ext}_{R}^{j}\left(R / \mathfrak{b}, H_{\mathfrak{a}}^{n}(M)\right) \\
& \longrightarrow \operatorname{Ext}_{R}^{j}\left(R / \mathfrak{b}, H_{\left(x_{1}, \ldots, x_{n}\right)}^{n}(M)\right) .
\end{aligned}
$$

Both end terms are finitely generated, so $\operatorname{Ext}_{R}^{j}\left(R / \mathfrak{b}, H_{\mathfrak{a}}^{n}(M)\right)$ is also finitely generated, as required.

Remark 3.4. Although Theorem 2.1 in $[\underline{6}$ is true, the proof of it holds some flaws. In the proof of Theorem 2.1 in [6], the $R$-module $N=E(M) / M$ is not necessarily finitely generated, where $E(M)$ is the injective hull of $M$. So, in the inductive hypotheses, we cannot assume that $M$ is finitely generated. Also, the assumption that $M$ is an $R$-module such that $\operatorname{Ext}_{R}^{i}(R / \mathfrak{b}, M)$ is a finitely generated $R$-module for every $i \leqslant s$, does not assert that $\Gamma_{\mathfrak{a}}(M)$ is $\mathfrak{a}$-cofinite (see [14, Corollary 1.8]).

The first part of the following corollary establishes the statement of Theorem 2.1 in [6]. The second part is the main result of [7].

Corollary 3.5 (Compare [7, Theorem A]). Let $\mathfrak{a}$ and $\mathfrak{b}$ be ideals of $R$ with $\mathfrak{a} \subseteq \mathfrak{b}$. Let $n$ be a non-negative integer such that $\operatorname{Ext}_{R}^{j}\left(R / \mathfrak{b}, H_{\mathfrak{a}}^{i}(M)\right)$ is finitely generated for all $i<n$ and all $j \in \mathbb{N}_{0}$. Then

(i) $\operatorname{Hom}_{R}\left(R / \mathfrak{b}, H_{\mathfrak{a}}^{n}(M)\right)$ is finitely generated, and so $\operatorname{Ass}_{R}\left(H_{\mathfrak{a}}^{n}(M)\right) \cap V(\mathfrak{b})$ is finite, and

(ii) $\operatorname{Ext}_{R}^{1}\left(R / \mathfrak{b}, H_{\mathfrak{a}}^{n}(M)\right)$ is finitely generated.

Proof. (i) Apply Theorem 3.3 with $j=0$ and note that $\operatorname{Ass}_{R}\left(\operatorname{Hom}_{R}\left(R / \mathfrak{b}, H_{\mathfrak{a}}^{n}(M)\right)\right)$ $=\operatorname{Ass}_{R}\left(H_{\mathfrak{a}}^{n}(M)\right) \cap V(\mathfrak{b})$.

(ii) Apply Theorem 3.3 with $j=1$.

Note that the first part of Corollary 3.5 is a generalization of the main results of $[2$ and $[12$.

Asadollahi and Schenzel, in [1, Theorem 1.2], by using spectral sequences, proved that over a local ring $(R, \mathfrak{m})$ if $M$ is a Cohen-Macaulay $R$-module and $t=\operatorname{grade}(\mathfrak{a}, M)$, then $\operatorname{Ext}_{R}^{2}\left(R / \mathfrak{a}, H_{\mathfrak{a}}^{t}(M)\right)$ is finitely generated if and only if $\operatorname{Hom}_{R}\left(R / \mathfrak{a}, H_{\mathfrak{a}}^{t+1}(M)\right)$ is finitely generated. The following corollary which is a generalization of 1 , Theorem $1.2]$ is a main result of 7 . 
Corollary 3.6 (Compare [7, Theorem B]). Let $\mathfrak{a}$ and $\mathfrak{b}$ be ideals of $R$ with $\mathfrak{a} \subseteq \mathfrak{b}$. Let $s$ be a non-negative integer such that $\operatorname{Ext}_{R}^{j}\left(R / \mathfrak{b}, H_{\mathfrak{a}}^{i}(M)\right)$ is finitely generated for all $i<s$ and all $j \in \mathbb{N}_{0}$. Then the following statements are equivalent:

(i) $\operatorname{Hom}_{R}\left(R / \mathfrak{b}, H_{\mathfrak{a}}^{s+1}(M)\right)$ is finitely generated.

(ii) $\operatorname{Ext}_{R}^{2}\left(R / \mathfrak{b}, H_{\mathfrak{a}}^{s}(M)\right)$ is finitely generated.

Proof. (i) $\Longrightarrow$ (ii) Apply Theorem 3.3 with $j=2$ and $n=s$.

(ii) $\Longrightarrow$ (i) Apply Theorem 3.3 with $j=0$ and $n=s+1$.

Delfino and Marley, in [5], proved that, over a local ring $R, H_{\mathfrak{a}}^{d}(M)$ is a-cofinite, where $d=\operatorname{dim} M$. In the following corollary, for a fixed integer $j$, we investigate the finiteness of $\operatorname{Ext}_{R}^{j}\left(R / \mathfrak{a}, H_{\mathfrak{a}}^{d-1}(M)\right)$.

Corollary 3.7. Let $(R, \mathfrak{m})$ be a Noetherian local ring, $\mathfrak{a}$ an ideal of $R$ and $M a$ finitely generated $R$-module of dimension $d$. Let $j$ be a non-negative integer such that $\operatorname{Ext}_{R}^{j+t+1}\left(R / \mathfrak{a}, H_{\mathfrak{a}}^{d-t-1}(M)\right)$ is finitely generated for all $t=1, \ldots, d-1$. Then $\operatorname{Ext}_{R}^{j}\left(R / \mathfrak{a}, H_{\mathfrak{a}}^{d-1}(M)\right)$ is finitely generated.

Proof. By [5, Theorem 3], $H_{\mathfrak{a}}^{d}(M)$ is a-cofinite. The result now follows from Theorem 3.3.

By using Theorem 3.3, in conjunction with [3, Corollary 3.3.3], we have the following corollary.

Corollary 3.8. Let $\mathfrak{b}$ be an ideal of $R$. Fix $j \in \mathbb{N}_{0}$ and $x, y \in \mathfrak{b}$. Then the following statements are equivalent:

(i) $\operatorname{Ext}_{R}^{j}\left(R / \mathfrak{b}, H_{(x, y)}^{2}(M)\right)$ is finitely generated.

(ii) $\operatorname{Ext}_{R}^{j+2}\left(R / \mathfrak{b}, H_{(x, y)}^{1}(M)\right)$ is finitely generated.

Marley and Vassilev, in [13, by using the Grothendieck spectral sequence established the following corollary in the case $\mathfrak{a}=\mathfrak{b}$. Now, Theorem 3.3 provides a slight generalization of [13, Proposition 2.5].

Corollary 3.9. Let $\mathfrak{a}$ and $\mathfrak{b}$ be ideals of $R$ with $\mathfrak{a} \subseteq \mathfrak{b}$. Suppose there exists an integer $n \in \mathbb{N}_{0}$ such that $\operatorname{Ext}_{R}^{j}\left(R / \mathfrak{b}, H_{\mathfrak{a}}^{i}(M)\right)$ is finitely generated for all $i \neq n$ and all $j \in \mathbb{N}_{0}$. Then $\operatorname{Ext}_{R}^{j}\left(R / \mathfrak{b}, H_{\mathfrak{a}}^{n}(M)\right)$ is finitely generated for all $j$.

\section{Finiteness properties of torsion functors OF LOCAL COHOMOLOGY MODULES}

Let $\mathfrak{a}$ and $\mathfrak{b}$ be ideals of $R$ with $\mathfrak{a} \subseteq \mathfrak{b}$. In this section, for fixed integers $j$ and $n$, we study the finiteness of $\operatorname{Tor}_{j}^{R}\left(R / \mathfrak{b}, H_{\mathfrak{a}}^{n}(M)\right)$. Using Proposition 2.3 together with straightforward modification to the arguments in the proof of Theorem 3.3, one can obtain the following theorem.

Theorem 4.1. Fix $j \in \mathbb{N}_{0}, n \in \mathbb{N}$, the ideals $\mathfrak{a}$ and $\mathfrak{b}$ with $\mathfrak{a} \subseteq \mathfrak{b}$, and a finitely generated $R$-module $M$ of dimension d. Assume that

(i) $\operatorname{Tor}_{j-t-1}^{R}\left(R / \mathfrak{b}, H_{\mathfrak{a}}^{n-t}(M)\right)$ is finitely generated for all $t=1, \ldots, n$, and

(ii) $\operatorname{Tor}_{j+k+1}^{R}\left(R / \mathfrak{b}, H_{\mathfrak{a}}^{n+k}(M)\right)$ is finitely generated for all $k=1, \ldots, d-n$.

Then $\operatorname{Tor}_{j}^{R}\left(R / \mathfrak{b}, H_{\mathfrak{a}}^{n}(M)\right)$ is finitely generated. 
Definition 4.2 (See [18). Let $(R, \mathfrak{m})$ be a local ring, $M$ an $R$-module and $E:=$ $E_{R}(R / \mathfrak{m})$ the injective hull of $R / \mathfrak{m}$. We define a prime ideal $\mathfrak{p}$ of $R$ to be a coassociated prime of $M$ if $\mathfrak{p}$ is an associated prime of $\operatorname{Hom}_{R}(M, E)$. We denote the set of coassociated primes of $M$ by $\operatorname{Coass}_{R} M$ (or $\operatorname{simply} \operatorname{Coass} M$, if there is no ambiguity about the underlying ring).

Note that Coass $M=\emptyset$ if and only if $M=0$. Also, for a finitely generated $R$-module $M$ and arbitrary $R$-module $N$, by [17, Theorem 1.22] and [5, Remark p. 50], we have that $\operatorname{Coass}_{R}\left(M \otimes_{R} N\right)=\operatorname{Supp}_{R} M \cap \operatorname{Coass}_{R} N$. In the following corollary, we provide a dual version of Corollary 3.5 "in some sense".

Corollary 4.3. Let $\mathfrak{a}$ and $\mathfrak{b}$ be ideals of $R$ with $\mathfrak{a} \subseteq \mathfrak{b}$. Let $n$ be a non-negative integer such that $\operatorname{Tor}_{j}^{R}\left(R / \mathfrak{b}, H_{\mathfrak{a}}^{i}(M)\right)$ is finitely generated for all $i>n$ and all $j \in \mathbb{N}_{0}$. Then

(i) $R / \mathfrak{b} \otimes_{R} H_{\mathfrak{a}}^{n}(M)$ is finitely generated and so, whenever $R$ is local, the set $V(\mathfrak{b}) \cap \operatorname{Coass}_{R}\left(H_{\mathfrak{a}}^{n}(M)\right)$ is finite, and

(ii) $\operatorname{Tor}_{1}^{R}\left(R / \mathfrak{b}, H_{\mathfrak{a}}^{n}(M)\right)$ is finitely generated.

For an $R$-module $M$, the cohomological dimension of $M$ with respect to $\mathfrak{a}$ is defined as

$$
\operatorname{cd}(\mathfrak{a}, M):=\max \left\{i \in \mathbb{Z}, H_{\mathfrak{a}}^{i}(M) \neq 0\right\} .
$$

Now, it follows from Corollary 4.3 that, for any ideals $\mathfrak{a}$ and $\mathfrak{b}$ with $\mathfrak{a} \subseteq \mathfrak{b}$, the $R$-modules $R / \mathfrak{b} \otimes_{R} H_{\mathfrak{a}}^{h}(M)$ and $\operatorname{Tor}_{1}^{R}\left(R / \mathfrak{b}, H_{\mathfrak{a}}^{h}(M)\right)$ are finitely generated, where $h$ is the cohomological dimension of $M$ with respect to $\mathfrak{a}$.

Corollary 4.4. Let $\mathfrak{a}$ and $\mathfrak{b}$ be ideals of $R$ with $\mathfrak{a} \subseteq \mathfrak{b}$. Let $s$ be a non-negative integer such that $\operatorname{Tor}_{j}^{R}\left(R / \mathfrak{b}, H_{\mathfrak{a}}^{i}(M)\right)$ is finitely generated for all $i>s$ and all $j \in \mathbb{N}_{0}$. Then the following statements are equivalent:

(i) $R / \mathfrak{b} \otimes_{R} H_{\mathfrak{a}}^{s-1}(M)$ is finitely generated.

(ii) $\operatorname{Tor}_{2}^{R}\left(R / \mathfrak{b}, H_{\mathfrak{a}}^{s}(M)\right)$ is finitely generated.

Proof. (i) $\Longrightarrow$ (ii) Apply Theorem 4.1 with $j=2$ and $n=s$.

(ii) $\Longrightarrow$ (i) Apply Theorem 4.1 with $j=0$ and $n=s-1$.

The next corollary follows from Theorem 4.1 and [3, Corollary 3.3.3].

Corollary 4.5. Let $\mathfrak{b}$ be an ideal of $R$. Fix $j \in \mathbb{N}_{0}$ and $x, y \in \mathfrak{b}$. Then the following statements are equivalent:

(i) $\operatorname{Tor}_{j}^{R}\left(R / \mathfrak{b}, H_{(x, y)}^{2}(M)\right)$ is finitely generated.

(ii) $\operatorname{Tor}_{j-2}^{R}\left(R / \mathfrak{b}, H_{(x, y)}^{1}(M)\right)$ is finitely generated.

Corollary 4.6. Let $\mathfrak{a}$ and $\mathfrak{b}$ be ideals of $R$ with $\mathfrak{a} \subseteq \mathfrak{b}$. Suppose there exists an integer $n \in \mathbb{N}_{0}$ such that $\operatorname{Tor}_{j}^{R}\left(R / \mathfrak{b}, H_{\mathfrak{a}}^{i}(M)\right)$ is finitely generated for all $i, j \in \mathbb{N}_{0}$ with $i \neq n$. Then $\operatorname{Tor}_{j}^{R}\left(R / \mathfrak{b}, H_{\mathfrak{a}}^{n}(M)\right)$ is finitely generated for all $j$.

Proof. It is immediate from Theorem 4.1.

We note an easy consequence of Corollary 4.6.

Corollary 4.7. Let $\mathfrak{a}$ and $\mathfrak{b}$ be ideals of $R$ with $\mathfrak{a} \subseteq \mathfrak{b}$ and $h \in \mathbb{Z}$. Suppose $\operatorname{Tor}_{j}^{R}\left(R / \mathfrak{b}, H_{\mathfrak{a}}^{i}(M)\right)$ is finitely generated for all $j \in \mathbb{N}_{0}$ and all $i<h$ and $H_{\mathfrak{a}}^{i}(M)=0$ for all $i>h$. Then $\operatorname{Tor}_{j}^{R}\left(R / \mathfrak{b}, H_{\mathfrak{a}}^{i}(M)\right)$ is finitely generated for all $i$ and $j$. In particular, if $H_{\mathfrak{a}}^{i}(M)=0$ for all $i>1$, then $\operatorname{Tor}_{j}^{R}\left(R / \mathfrak{b}, H_{\mathfrak{a}}^{i}(M)\right)$ is finitely generated for all $i$ and $j$. 


\section{ACKNOWLEDGMENTS}

The author is deeply grateful to the referee for careful reading of the manuscript and for the helpful suggestions.

\section{REFERENCES}

[1] Asadollahi, J.; Schenzel, P., Some results on associated primes of local cohomology modules, Japan. J. Math. (N.S.) 29 (2003), no. 2, 285-296. MR2035541 (2004m:13043)

[2] Brodmann, M. P.; Lashgari F. A., A finiteness result for associated primes of local cohomology modules, Proc. Amer. Math. Soc. 128 (2000), no. 10, 2851-2853. MR1664309 (2000m:13028)

[3] Brodmann, M.; Sharp, R.Y., 'Local cohomology - an algebraic introduction with geometric applications', Cambridge Studies in Advanced Mathematics No. 60, Cambridge University Press (1998). MR.1613627 (99h:13020)

[4] Delfino, D., On the cofiniteness of local cohomology modules, Math. Proc. Cambridge Philos. Soc. 115 (1994), no. 1, 79-84. MR:1253283 (94m:13023)

[5] Delfino, D; Marley, T., Cofinite modules and local cohomology, J. Pure Appl. Algebra 121 (1997), no. 1, 45-52. MR1471123 (98g:13015)

[6] Dibaei, M. T.; Yassemi, S., Associated primes and cofiniteness of local cohomology modules, Manuscripta Math. 117 (2005), no. 2, 199-205. MR2150481

[7] Dibaei, M. T.; Yassemi, S., Finiteness of extension functors of local cohomology modules, preprint (arXiv: math. AC/0509340 V1 15 Sep. 2005).

[8] Grothendieck, A., Cohomologie locale des faisceaux cohérents et théorèmes de Lefschetz locaux et globaux (SGA 2), North-Holland Publishing Co., Amsterdam (1968). MR0476737 $(57: 16294)$

[9] Hartshorne, R., Affine duality and cofiniteness, Invent. Math. 9 (1969/1970), 145-164. MR0257096 (41:1750)

[10] Huneke, C; KoH, J., Cofiniteness and vanishing of local cohomology modules, Math. Proc. Cambridge Philos. Soc. 110 (1991), no. 3, 421-429. MR1120477 (92g:13021)

[11] Khashyarmanesh, K.; Salarian, Sh., Filter regular sequences and the finiteness of local cohomology modules, Comm. in Algebra 26(8) (1998), 2483-2490. MR1627876 (99h:13021)

[12] Khashyarmanesh, K.; Salarian, Sh., On the associated primes of local cohomology modules, Comm. in Algebra 27 (1999), no. 12, 6191-6198. MR1726302 (2000m:13029)

[13] Marley, T.; Vassilev, J. C., Cofiniteness and associated primes of local cohomology modules, J. Algebra 256 (2002), no. 1, 180-193. MR.1936885 (2003j:13025)

[14] Melkersson, L., Properties of cofinite modules and applications to local cohomology, Math. Proc. Cambridge Philos. Soc. 125 (1999), no. 3, 417-423. MR.1656785 (99k:13024)

[15] Schenzel, P.; Trung, N. V.; Cuong, N. T., Verallgemeinerte Cohen-Macaulay-Moduln, Math. Nachr. 85 (1978), 57-73. MR0517641 (80i:13008)

[16] Stǘckrad, J.; Vogel, W., 'Buchsbaum rings and applications', VEB Deutscher Verlag der Wissenschaftan, Berlin (1986). MR0881220 (88h:13011a)

[17] Vasconcelos, W. V., 'Divisor theory in module categories', North-Holland Mathematics Studies, No. 14, North-Holland, Amsterdam (1974). MR0498530 (58:16637)

[18] Yassemi, S., Coassociated primes, Comm. in Algebra 23 (1995), no. 4, 1473-1498. MR:1317409 (96e:13003)

[19] YoshidA, K.-I., Cofiniteness of local cohomology modules for ideals of dimension one, Nagoya Math. J. 147 (1997), 179-191. MR1475172 (98j:13014)

Department of Mathematics, Mashhad University, P.O. Box 1159-91775, Mashhad, Iran; Institute for Studies in Theoretical Physics and Mathematics, P.O. Box 193955746, TEHran, Iran

E-mail address: Khashyar@ipm.ir 